\title{
Absolute Magnitude Test: Testing Cosmological Models Based on Compilations of Supernovae SNe Ia "Union" and "Union2"
}

\author{
Abraham P. Mahtessian*, Garen S. Karapetian, Vardan H. Movsisyan and Lazar A. Mahtessian \\ NAS RA V. Ambartsumian Byurakan Astrophysical Observatory (BAO), Armenia \\ Email: amahtes@bao.sci.am
}

\begin{abstract}
In the present work, on the basis of compilations of supernovae Sne Ia Union and Union2, two cosmological models describing the Universe are considered. One of them (a model with a cosmological constant of zero) was widespread until 1998 when a study of type Ia supernovae showed that the Universe is expanding at an accelerating rate. After that, a model for the case of a flat universe, which is also discussed in this paper, received great recognition.

To accept or reject any cosmological model, it is proposed to carry out a test based on the statement that the absolute magnitude of type Ia supernovae should not depend on the redshift $\left(M_{S N e I a} \neq M_{S N e I a}(z)\right)$, i.e. these supernovae are distance indicators. (This test is especially important when own software is used to analyze the Hubble diagram, as was done for example by Kowalski et al. 2008 and Amanullah et al. 2010.)

It turns out that the values of the model parameters obtained according to this principle are also confirmed by the Hubble diagram. It is with these data that the best approximation of the observed data is obtained. Mathematical analysis of the Hubble diagram speaks in favor of a model with a cosmological constant of zero. This model also has one important advantage: it does not need to accept the idea of such a hypothetical substance as "Dark Energy".

Thus, it turns out that we are in open space, filled to about $30 \%$ of the critical density of matter, which participates in gravitational interaction and expands with deceleration. This value coincides with the accepted amount of visible and dark matter.

If all the same to accept the model of flat universe, then we must abandon the superiority of energy over mass $\left(\Omega_{\Lambda}<0.5\right)$.
\end{abstract}

Keywords: Cosmological models, supernovae, absolute magnitude test, dark energy, dark matter: general.

\section{Introduction}

A type Ia supernova is believed to form when a white dwarf captures matter from its neighbor in a binary system, as a result of which its mass increases to a possible limit - the Chandrasekhar limit, when degraded electrons cannot resist gravitational pressure and the star passes into an unstable stage. An increase in the temperature and density of the star makes it possible to transform carbon and oxygen into ${ }^{56} \mathrm{Ni}$, which is accompanied by a thermonuclear explosion (Fowler and Hoyle 1960). The brightness of the star increases so much that sometimes it exceeds the brightness of the host galaxy, and it can be seen throughout a few thousand megaparsecs. The mass of an exploded star is always near the Chandrasekhar limit, therefore, in the case of such explosions, the absolute magnitude of the star may vary only within small limits. This allows these supernovae to be used as ideal indicators of distance (Sandage and Tammann 1982).

This feature of type Ia supernovae allows studying the behavior of the Universe at considerable distances and evaluating the truth of a particular cosmological model.

One of the methods for determining the distance between extragalactic objects is Hubble's law.

At the beginning of the 20th century, Hubble obtained a result, which led to the conclusion that the Universe is expanding, and that the speed of this expansion is directly proportional to the distance from the observer. Hubble's work is based on the fact, discovered by Slipher, that the spectral lines in the spectra of galaxies are shifted towards the long part of the wave (Slipher 1924). Hubble discovered that this shift increases with increasing distance to galaxies (Hubble, 1929).

Another method for determining distance is based on the distance modulus.

$$
M=m-5 \lg D_{L}-25,
$$


where $m$ is the apparent magnitude, $M$ is the absolute magnitude, and $D_{L}$ is the luminosity distance. When calculating the distance by this method, it is necessary to accurately estimate the value of the apparent magnitude of the object (take into account the galactic absorption, K-correction, spectral region, etc.). The absolute magnitude should be known either from theoretical approaches (for example, for stars of type Ia supernovae) or from empirical dependencies (for example, in the case of Cepheids).

Riess et al. (1998) and Perlmutter et al. (1999), to study the properties of the universe, made two assumptions:

a. Those Ia-type supernovae are distance indicators, that is, their absolute magnitude values can be considered constant.

b. That the Friedmann-Robertson-Walker (FRW) cosmological model for the case of a flat universe accurately describes the Universe.

Taking into account fairly accurately the phenomena that can affect the result, calculating the apparent magnitudes, they compared them with the values obtained from the cosmological model. It turned out that the apparent magnitude was weaker than that obtained from the theory, that is, these objects are farther than they could be based on the Hubble law. This led to the idea that the universe is expanding with acceleration. In this regard, the idea of "dark energy" was introduced.

In order to avoid the idea of dark energy, various attempts were made to explain the discrepancy between the theoretical and observed luminosities of supernovae by other phenomena. Let's list some of them.

a. The weakening of the apparent magnitude of a star is due to the absorption or scattering of light by matter in the path of light.

b. There is an evolution of the luminosity of a white dwarf, depending on the chemical composition of the host galaxy over time.

c. Gravity lenses.

d. The reason is the uneven distribution of matter in the universe.

e. It is assumed that in nature there are two types of supernovae Ia. The second type is not numerous and is formed from the merger of two white dwarfs. As a result of the merger, the mass of the exploding star is no longer fixed.

f. Observational errors may also increase due to the fact that the brightness curves of various supernovae are recorded in different conditions (on Earth and in space).

The degree of influence of these phenomena has been discussed in various studies showing that many of these inaccuracies cannot be considered satisfactory to refute the results obtained by Riess et al. (1998) and Perlmutter et al. (1999). They can be found in Weinberg (2008).

In the studies of Riess et al. (1998) and Perlmutter et al. (1999), the processing of observational material was performed quite carefully and the refined apparent magnitudes are beyond doubt. Despite this, further work was carried out to refine the results obtained.

In this paper, we discuss two models: the $\Lambda \mathrm{CDM}$ model (also known as the Friedmann-RobertsonWalker cosmology) used by Riess et al. (1998) and Perlmutter et al. (1999) for the case of a flat universe $\left(\Omega_{K}=0\right)$ and a model with zero cosmological constant which was widely used before this work (until 1999). The first model assumes the existence of dark energy; in the second model, this hypothesis is not necessary.

In the case of $\Lambda C D M$ universe, the dependence of the luminosity distance on the redshift is determined by the following formula:

$$
D_{L}=C H_{0}^{-1}(1+z)\left|\Omega_{K}\right|^{-1 / 2} \times \operatorname{sinn}\left\{\left|\Omega_{K}\right|^{1 / 2} \int_{0}^{z} d z\left[(1+z)^{2}\left(1+\Omega_{M} z\right)-z(2+z) \Omega_{\Lambda}\right]^{-1 / 2}\right\}
$$

where $z$ is the redshift of the object. $\Omega_{K}$ is associated with the curvature of space and in the case of flat universe it is 0 (Carroll et al., 1992): $\Omega_{K}=1-\Omega_{M}-\Omega_{\Lambda}$, sinn $=\sinh$, when $\Omega_{K} \geq 0$ and $\operatorname{sinn}=\sin$, when $\Omega_{K} \leq 0$. In the case of $\Omega_{K}=0$, we will have:

$$
D_{L}=\frac{C(1+z)}{H_{0}} \int_{0}^{z} d z\left[(1+z)^{2}\left(1+\Omega_{M} z\right)-z(2+z) \Omega_{\Lambda}\right]^{-1 / 2}
$$


or

$$
D_{L}=\frac{C(1+z)}{H_{0}} \int_{0}^{z} d z\left[(1+z)^{3} \Omega_{M}+\Omega_{\Lambda}\right]^{-1 / 2}
$$

If we assume that $\Omega_{\Lambda}=1$, and $\Omega_{M}=0$, we will have (Weinberg, 2008)

$$
D_{L}=\frac{C}{H_{0}}\left(z+z^{2}\right)
$$

If $\Omega_{\Lambda}=0$, and $\Omega_{M}=1$, we have

$$
D_{L}=\frac{2 C}{H_{0}}[(1+z)-\sqrt{1+z}]
$$

It should be noted that in 1998, before the work of Riess et al. (1998) and Perlmutter et al. (1999) the equations of the general theory of relativity (GTR) with the zero cosmological constant $\left(\Omega_{\Lambda}=0\right)$ were commonly used. Using this model, Mattig (1958) precisely integrated these equations and obtained the luminosity distance depending on the redshift.

$$
D_{L}=\frac{C}{H_{0} q_{0}^{2}}\left[q_{0} z+\left(q_{0}-1\right)\left(\sqrt{1+2 q_{0} z}-1\right)\right]
$$

where $q_{0}$ is the deceleration parameter, in this case:

$$
q_{0}=\frac{\Omega_{M}}{2}
$$

(6) with $q_{0}=0.5$ coinciding with (5).

For the luminosity distance in the flat universe, we will use formula (3). For the luminosity distance in the model with $\Omega_{\Lambda}=0$, we will use formula (6).

\section{Test}

Our approach is as follows: if somehow the calculated luminosity distances are accurate and the obtained apparent magnitudes are also accurate, then the absolute magnitudes obtained using these values and formula (1) should not contradict the assumption that Type Ia Supernovae are distance indicators, i.e. the values of the absolute magnitudes of these supernovae should not depend on the redshift. Let us call this method the test of absolute magnitudes and mathematically denote it as follows: $M_{S N e I a} \neq M_{S N e I a}(z)$. (The idea of the existence of the luminosity evolution of type Ia supernovae is often touched upon in the literature. If we assume that we have an observational or theoretical justification for this idea, then the absolute magnitude test should be written in the form $M_{S N e I a}=f(z)$, where the function $f$ is the evolutionary dependence of the absolute magnitude of the supernova at the maximum brightness on the red shift).

When talking about standard candles, we believe that this test is more important than the Hubble diagram. But, as we will see later, these two methods give a consistent result.

As mentioned above, the adjusted apparent magnitudes of supernovae in Riess et al. (1998) and Perlmutter et al. (1999) and in the subsequent works, do not raise doubts. Only distance estimates remain, which are closely related to the chosen cosmological model.

\section{Samples}

As the first sample of type Ia supernovae, we will use a compilation made by Kowalski et al. (2008) from several samples (SNe Ia "Union"; Kowalski +, 2008; http://vizier.u-strasbg.fr/viz-bin/VizieR-3?source $=\mathrm{J} / \mathrm{ApJ} / 686 / 749)$. The sample consists of 414 stars, for which apparent magnitudes are given in Vizier (the others have "bad" brightness curves) that we used for our work. This "Compilation" includes supernovae taken from various papers (Hamuy et al. 1996, Krisciunas et al. 2004a, 2004b, Riess et al. 1999, Jha et al. 2006, Kowalski et al. 2008, Riess et al. 1998, Garnavich et al. 1998, Schmidt et al. 1998, 
Perlmutter et al. 1999, Tonry et al. 2003, Barris et al. 2004, Knop et al. 2003, Riess et al. 2007, Astier et al. 2006, Miknaitis et al. 2007). The principles of combining these samples are well described in their article.

The second sample we use is the SNIa "Union2" sample (Amanullah et al. 2010; http://vizier.ustrasbg.fr/viz-bin/VizieR-3?-source=J/ApJ/716/712). The sample consists of 719 supernovae mentioned in 17 papers (Hamuy et al. 1996; Krisciunas et al. 2005; Riess et al. 1999; Jha et al. 2006; Kowalski et al. 2008; Hicken et al. 2009a, 2009b; Holtzman et al 2008; Riess et al. 1998; Perlmutter et al. 1999; Barris et al. 2004; Amanullah et al. 2008; Knop et al. 2003; Astier et al. 2006; Miknaitis et al. 2007; Tonry et al. 2003; Riess et al. 2007; Amanullah et al. 2010).

All the principles used in the SNIa "Union" sampling were observed and one more correction was added - the dependence of the absolute luminosity of a supernova on the luminosity of the host galaxy.

Figure 1 shows the Hubble diagram for the SNIa "Union" sample stars.

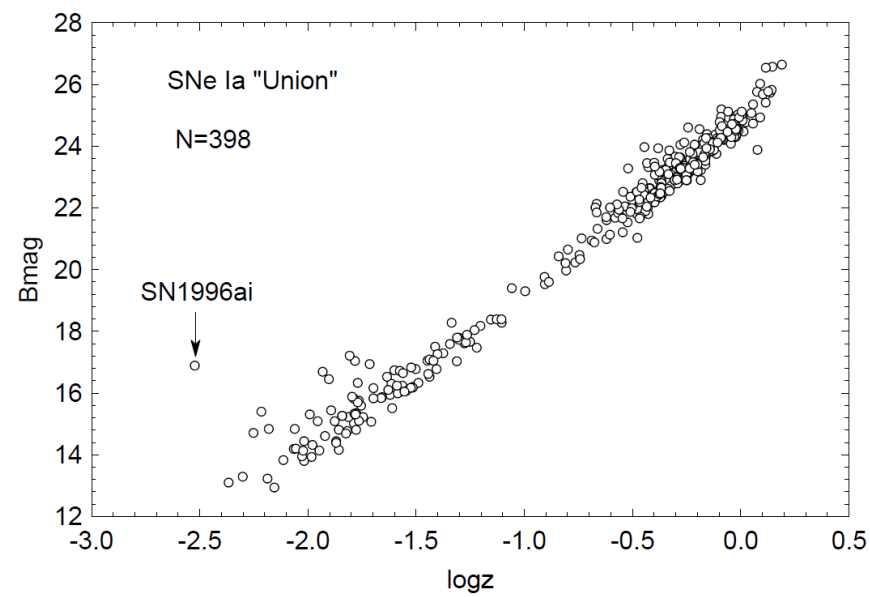

Figure 1. Hubble diagram for SNIa "Union" sample stars.

Based on the fact that SN1996ai strongly deviates from the regularity, we excluded it from further study. Thus, 397 stars remained in the "Union" sample. So that we can be completely independent of any cosmological model, we will initially carry out statistics with these 397 stars. Then we look at the sample given in Kowalski et al. (2008).

We will look at the Union2 sample for several cases.

1. In the sample we include all the stars of the SNeIa Union2 sample with luminosity data, with the exception of the stars 1996ai, 2003fa, 2005ew, 2006bk, 2006mq, which clearly fall out of the general Hubble diagram.

2. Consider exactly the sample that was used in Amanullah et al. (2010).

3. Reduce the width of the Hubble diagram to 1 magnitude, with the exception of regional stars. We will try to do this regardless of cosmological models. The procedure for excluding these stars is described below.

4. In sample 2, we also exclude the stars indicated in Amanullah et al. (2010) in the "Fail" column.

\section{Results}

\subsection{SNIa "Union"}

Figure 2a shows the absolute magnitudes of Ia-type supernovae calculated for luminosity distances obtained within the flat, $\Lambda C D M$ universe, depending on the redshift for the case of $\Omega_{\Lambda}=1$ and $\Omega_{M}=0$.

Figure $2 \mathrm{~b}$ shows the absolute magnitudes of Ia-type supernovae calculated for luminosity distances obtained in the flat, $\Lambda C D M$ universe framework, depending on the redshift for the case of $\Omega_{\Lambda}=0.7$ and $\Omega_{M}=0.3$ 
Figure 2c shows the absolute magnitudes of Ia-type supernovae, obtained taking into account the luminosity distances calculated in the framework of cosmology with a cosmological constant of zero $\left(\Omega_{\Lambda}=0\right)$, according to formula (6), depending on the redshift for the case $q_{0}=0.5\left(\Omega_{M}=1\right)$.

Figure 2d shows the absolute magnitudes of Ia-type supernovae, obtained while taking into account the luminosity distances calculated by the formula (6), depending on the redshift for the case $q_{0}=0.05$.
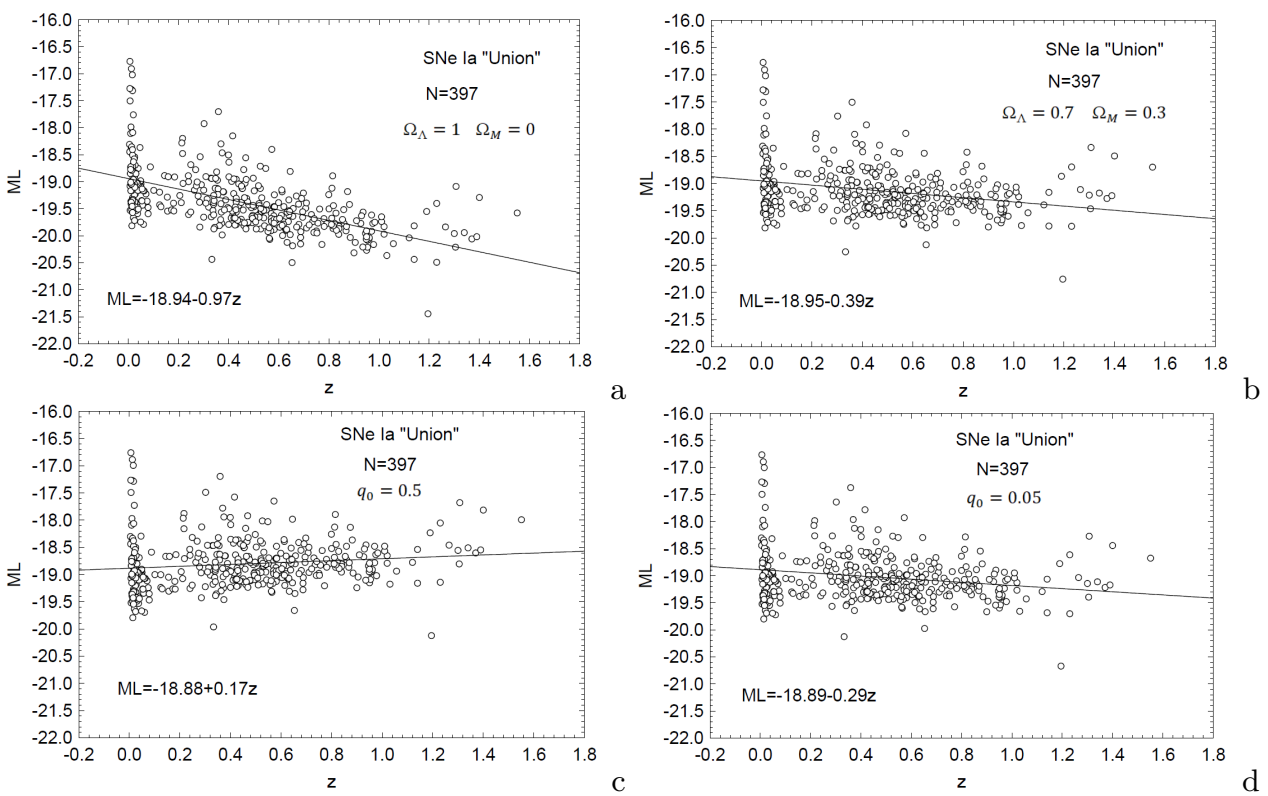

Figure 2. The values of the absolute magnitudes of supernovae Ia, calculated for various cosmological models and the various values of their parameters, depending on the redshift.
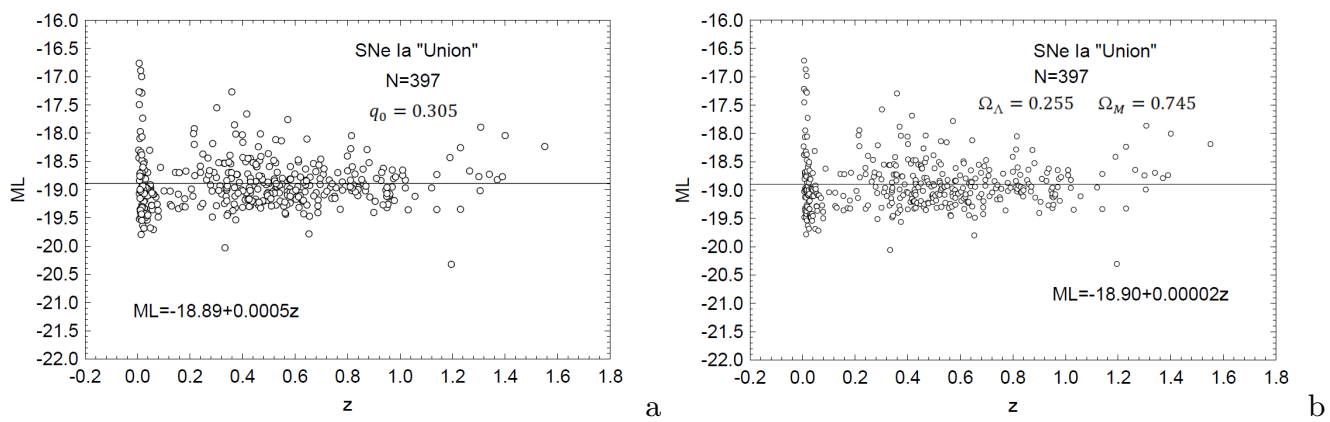

Figure 3. The values of the absolute magnitudes of the Ia-type supernova taking into account the luminosity distances calculated by formula (6), for the case $q_{0}=0.305$, and the luminosity distances calculated by formula (3), for the case $\Omega_{\Lambda}=0.255, \Omega_{M}=0.745$, depending on the redshift.

From Figure 2 it follows that in the case of the models and the values of their parameters discussed there the dependence of the absolute magnitude on the distance is obvious.

It is also obvious that in the case of a model for a flat, $\Lambda C D M$ universe, there is a value of $\Omega_{\Lambda}$ between 0 and 0.7, where the absolute magnitude of the star is almost independent of distance. And in the case of a model with a cosmological constant of zero, this value is in the range $q_{0}=0.05 \div 0.5$. A simple selection shows that in the case of a model with $\Omega_{\Lambda}=0$ and $q_{0} \sim 0.3$, the absolute magnitude does not depend on 
the redshift $\left.M_{S N e I a} \neq M_{S N e I a}(z)\right)$, and in the case of a flat universe model, this result is obtained with $\Omega_{\Lambda}=0.25, \Omega_{K}=0.75$.

Figure 3a shows the values of the absolute magnitudes of the Ia-type supernova taking into account the luminos ity distances calculated by formula (6), for the case $q_{0}=0.305$, and in Figure $3 \mathrm{~b}$ values of absolute magnitudes, taking into account the luminosity distances calculated by formula (3), for the case of $\Omega_{\Lambda}=0.255, \Omega_{M}=0.745$, depending on the redshift. In both cases, the lack of dependence is obvious.

Thus, the values of the absolute magnitudes of supernovae, based on the cosmological constant $\Omega_{\Lambda}=0$ with the deceleration parameter $q_{0}=0.305$, do not depend on redshift. In the case of a cosmological model with $\Omega_{K}=0$, this result is achieved for the values $\Omega_{\Lambda}=0.255, \Omega_{M}=0.745$. This means that in these cases we get a result that does not contradict the preliminary assumption (that Ia-type supernovae have a constant absolute magnitude). Below, we will see that this result is also confirmed by the Hubble diagram of type Ia supernovae.
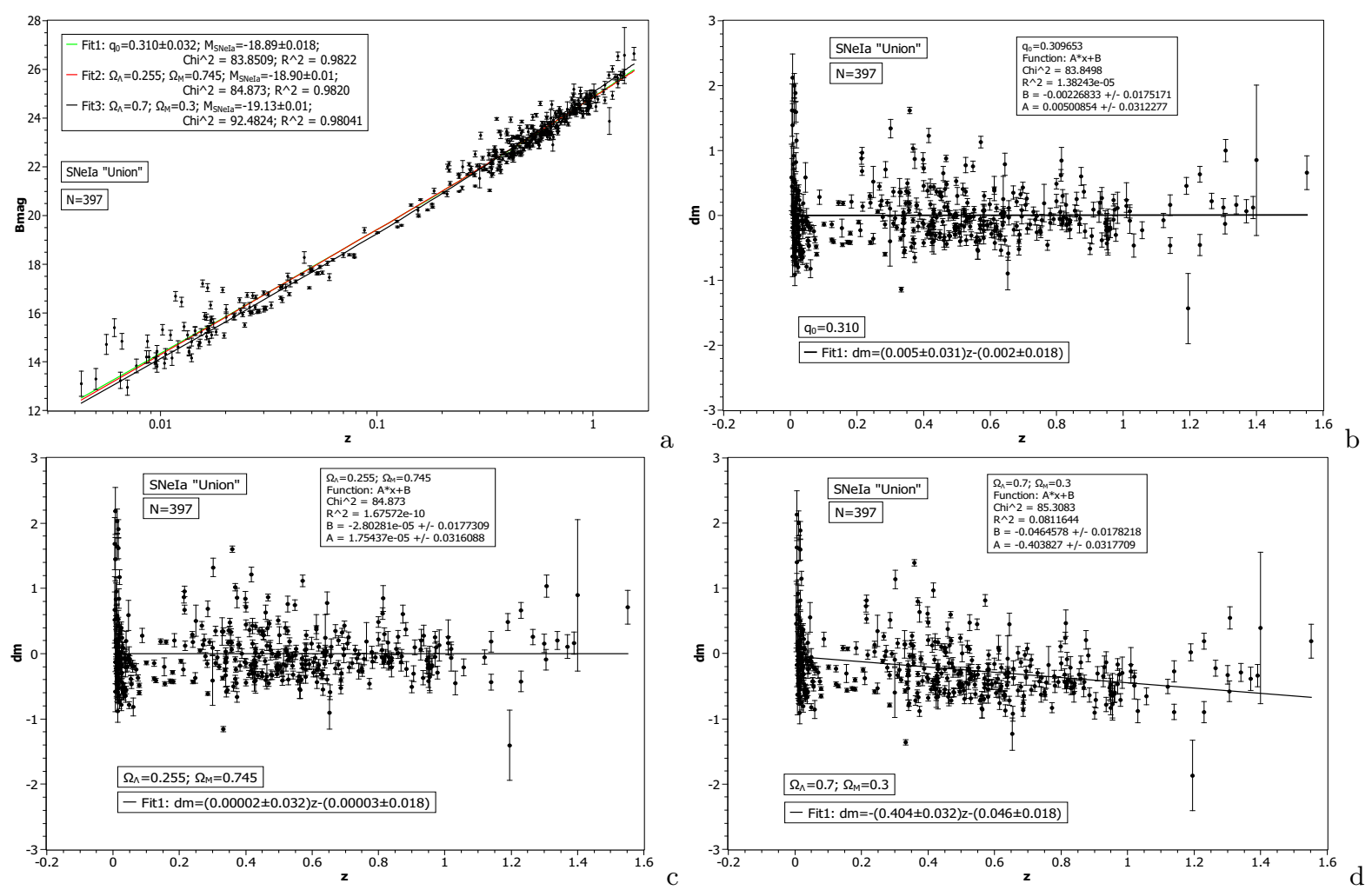

Figure 4. Hubble diagram for a sample of SNe Ia "Union" (a) and dependences of residuals on redshifts for different cosmological models (b, c, d).

Figure 4 shows the Hubble diagram for the SNeIa "Union" sample and its theoretical approximations for various cosmological models. The theoretical curves represent the dependency:

$$
B_{m a g}^{t h}(z)=M_{S N e I a}+5 \times \log D_{L}(z)+25
$$

where $B_{m a g}^{t h}(z)$ are the theoretical values of apparent magnitudes. Calculations are performed taking $H_{0}=67.74 \mathrm{~km} \cdot \mathrm{s}^{-1} \cdot \mathrm{Mpc}^{-1}$. The approximation is carried out through the program SciDAVIs (http://scidavis.sourceforge.net/).

For each model, we calculated $D_{L}(z)$, using formulas (3) and (6). In the case of a model with a cosmological constant of zero, the Hubble diagram is best approximated at $q_{0}=0.310 \pm 0.032$, $M_{S N e I a}=-18.89 \pm 0.018$, which fits very well with the result obtained from the test of absolute magnitudes 
(Figure 3), when we assumed that the model must not violate the rule $M_{S N e I a} \neq M_{S N e I a}(z)$. In the case of a cosmological model with $\Omega_{\Lambda}=0.255, \Omega_{M}=0.745$, it turns out that $M_{S N e I a}=-18.90 \pm 0.01$. The diagram also tested the model with $\Omega_{\Lambda}=0.7, \Omega_{M}=0.3$, where the approximation gave the value $M_{S N e I a}=-19.13 \pm 0.01$. As can be seen from Figure 4 , the sum of squared distances of observation points from the studied curves is the smallest in the case of a model with zero cosmological constant $\left(\Omega_{\Lambda}=0\right)$, and for $\Omega_{K}=0$, the values of $\Omega_{\Lambda}=0.255, \Omega_{M}=0.745$ represent the curve much better than $\Omega_{\Lambda}=0.7, \Omega_{M}=0.3$. Along with the Hubble diagram, Figure 4 also shows the dependence of residuals

$$
d m=B_{m a g}-B_{m a g}^{t h}
$$

from redshift.

As can be seen, these residues are most independent of the redshift in the case of a model with a cosmological constant of zero $\left(\Omega_{\Lambda}=0\right)$ with $q_{0}=0.310$, and in the case of a model $\Omega_{K}=0$ for $\Omega_{\Lambda}=$ $0.255, \Omega_{M}=0.745$ which should have been expected. In the case of a model with $\Omega_{\Lambda}=0.7, \Omega_{M}=0.3$, the dependence of the residuals on the redshift is obvious. These dependencies also show that the smallest quadratic deviations are obtained in the case of a zero cosmological constant.

Now let's discuss the sample examined in Kowalski et al. (2008). The sample is based on the SNe Ia "Union" sample with the removal of some "fail" stars. They are listed in the "Note" column in their table 11. The sample contains 307 stars:

In the case of a model with a zero cosmological constant $\left(\Omega_{\Lambda}=0\right)$, the test $M_{S N e I a} \neq M_{S N e I a}(z)$ gives the value $q_{0}=0.141$, and in the case of the model with $\Omega_{K}=0$, this test gives the values $\Omega_{\Lambda}=0.501, \Omega_{M}=0.499$. For these cases, the absolute magnitude - redshift relationship is shown in Figure 5. Figure 5 also shows this relationship for the values obtained in Kowalski et al. (2008), with $\Omega_{\Lambda}=0.713, \Omega_{M}=0.287$.
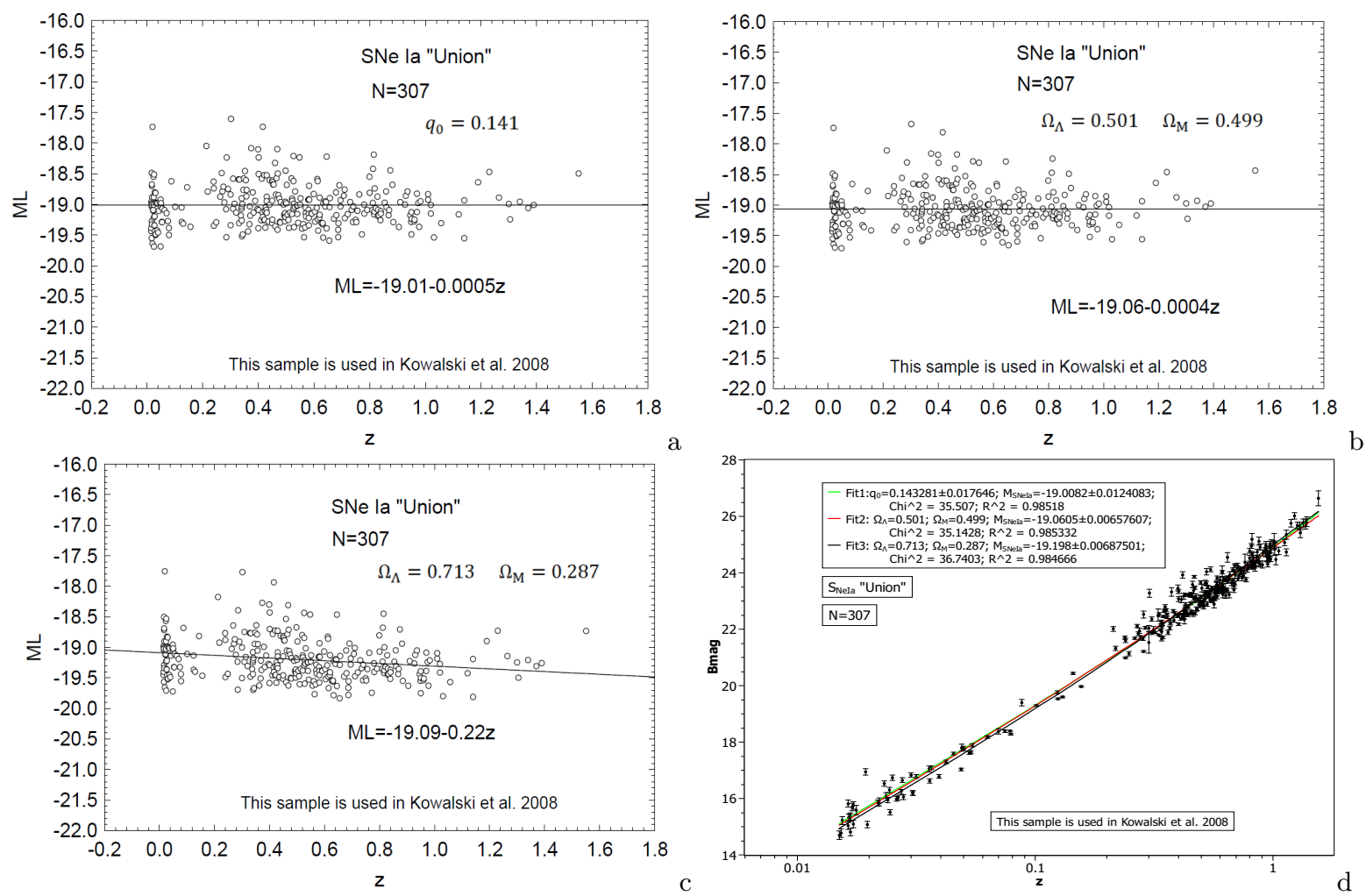

Figure 5. Dependence of absolute magnitude - redshift (a, b, c) and Hubble diagram (d) for the sample studied in Kowalski et al. (2008) for different parameters of the models under discussion. 
In the first two cases, there is no dependence, and in the third case, it is obvious. Figure 5d shows the Hubble diagram of this sample. Approximations of the Hubble diagram by the corresponding theoretical curves show that the first two cases represent the observed data better than the third one. Meanwhile, the $q_{0}$ and $M_{S N e I a}$ values obtained through the Hubble diagram, in the case of a model with a cosmological constant of zero, corresponding to the values obtained using the $M_{S N e I a} \neq M_{S N e I a}(z)$ test. And in the case of a model with $\Omega_{K}=0$, we made an approximation for fixed $\Omega_{\Lambda}$ and $\Omega_{M}$ values and estimated the value of the corresponding parameter $M_{S N e I a}$, which also corresponds to the test estimate $M_{S N e I a} \neq M_{S N e I a(z)}$.

Thus, the sample studied by Kowalski et al. (2008) in the case of a flat, $\Lambda C D M$ universe model, does not show the values obtained in the work $\left(\Omega_{\Lambda}=0.713, \Omega_{M}=0.287\right)$, but instead shows $\Omega_{\Lambda}=$ $0.501, \Omega_{M}=0.499$. Values $\Omega_{\Lambda}=0.713, \Omega_{M}=0.287$ are far from being considered the best approximation values.

Now consider these issues based on the SNIa "Union2" sample.

\subsection{SNIa "Union2"}

We perform the same actions with the SNIa "Union2" sample (Amanullah et al., 2010). Eliminating the stars with the "bad" luminosity curve in the sample, we leave 685 stars.

Figure 6 shows a Hubble diagram. In the beginning, let's try not to interfere strongly with the Hubble diagram. We remove from the sample only the stars that are strictly deviating from the general pattern and are marked on the diagram. In the sample remains 680 stars, on the basis of which we will conduct a preliminary study.

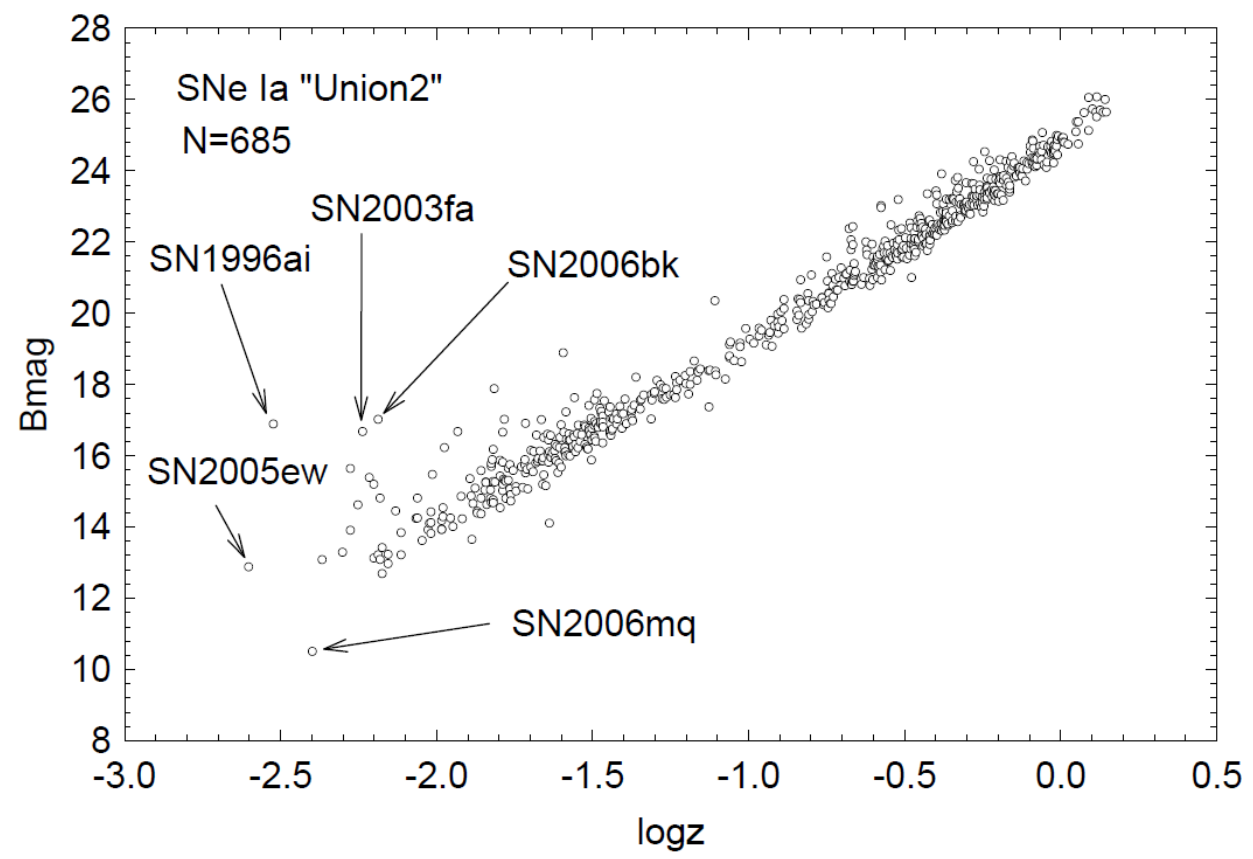

Figure 6. Hubble diagram for SNIa "Union2" sample.

Figure 7 shows the absolute magnitude - redshift dependence for the corresponding model for four cases.
a. $\Omega_{\Lambda}=1, \Omega_{M}=0$
b. $\Omega_{\Lambda}=0.7, \Omega_{M}=0.3$
c. $q_{0}=0.5\left(\right.$ or $\left.\Omega_{M}=1\right)$
d. $q_{0}=0.05$ 

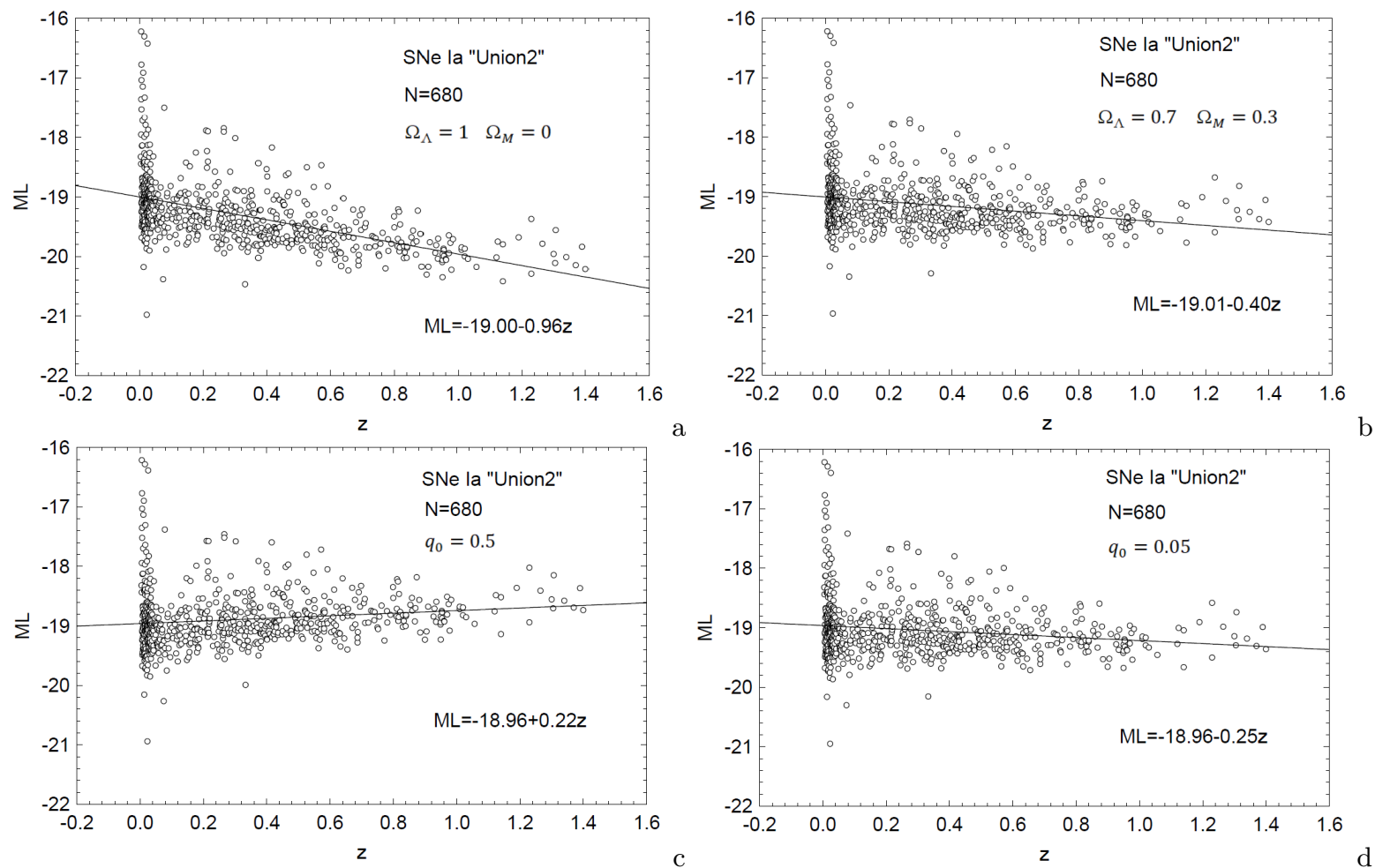

Figure 7. Dependence between absolute magnitude and redshift for four cases a $-\Omega_{\Lambda}=1, \Omega_{M}=0$; b $\Omega_{\Lambda}=0.7, \Omega_{M}=0.3 ; \mathrm{c}-q_{0}=0.5$ (or $\Omega_{M}=1$ ); $\mathrm{d}-q_{0}=0.05$ for SNIa "Union2" sample.

In the above four cases, the dependence of the absolute magnitude on the redshift is obvious. The figure also shows that in the region $\Omega_{\Lambda}=0.7, \quad \Omega_{M}=0.3$ and $\Omega_{\Lambda}=0.0, \quad \Omega_{M}=1.0\left(q_{0}=0.5\right)$ is the value $\left(\Omega_{\Lambda}, \Omega_{M}\right)$ at which the dependence of absolute magnitude - redshift will be insignificant. In the case of the model with $\Omega_{\Lambda}=0$, such an insignificant dependence can be found for $q_{0}$ in the range from $q_{0}=0.5$ to $q_{0}=0.05$.

Figure 8 shows these dependencies for models $q_{0}=0.265$ and $\Omega_{\Lambda}=0.3, \Omega_{M}=0.7$. It is clear that in these cases the dependence of the absolute magnitude on the redshift is insignificant.
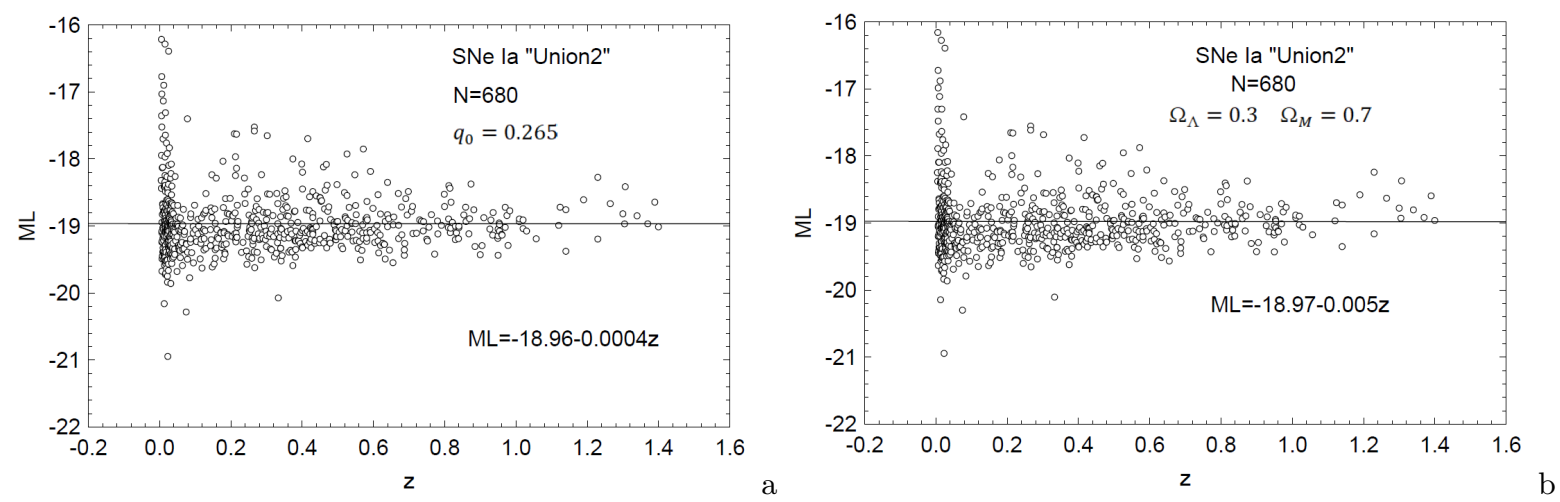

Figure 8. Dependence between absolute magnitude and redshift for models $q_{0}=0.265$ and $\Omega_{\Lambda}=0.3, \Omega_{M}=0.7$ for SNIa "Union2" sample 




Figure 9. Hubble diagram for SNIa "Union2" sample.

Now consider the Hubble diagram. This is shown in Figure 9. As in Figure 4, we also considered 3 cases here.

a. $\Omega_{\Lambda}=0.7, \Omega_{M}=0.3$ : in this case, using the least squares method, we get $M_{S} N e I a=-19.13 \pm 0.01$. We consider this case on the basis of the fact that most studies give similar values.

b. $\Omega_{\Lambda}=0.3, \Omega_{M}=0.7$ : in this case the approximation gives $M_{S} N e I a=-18.98 \pm 0.01$. We consider this case based on the fact that with these parameters there is no dependence between absolute magnitude and redshift.

c. In this case, the approximation gives $q_{0}=0.268 \pm 0.027, M_{S} N e I a=-18.96 \pm 0.01$. As can be seen, these values are consistent with Figure 8, that is, precisely at these values, we obtain the best approximation to the Hubble diagram, and the absolute magnitude does not depend on the redshift.

In order to save space, we do not give here the dependence of the residues on the redshift, but we can say that they are similar to the results of the SNeIa "Union" sample.

Thus, these graphs show that the squared deviations from the theoretical curve are the smallest in the case of the zero cosmological constant, and second: the obtained values of $q_{0}=0.268 \pm 0.027$ and $M_{\text {SNeIa }}=-18.96 \pm 0.012$, almost coincide with the values obtained in Figure 8 where it was only assumed that the absolute magnitude of the supernova should not depend on the redshift $\left(M_{S N e I a} \neq M_{S N e I a}(z)\right)$.

Here, the "Union2" sample was not particularly cleansed from stars that are far from the "distance indicators". This was done for the sole purpose of ensuring that the test we proposed was carried out without interfering with the Hubble diagram. Improper intervention in the Hubble diagram may give an advantage to a particular model depending on the filtering method. But, on the other hand, the inclusion of "fake" supernovae SNeIa can lead to inaccuracies in the estimation of cosmological parameter values.

Now let's examine the sample used in Amanullah et al. (2010). Following several principles, the authors, after clearing the sample, left 557 supernovae there. Using the principle proposed above, we found those values $\Omega_{\Lambda}, \Omega_{M}$ ) and $q_{0}$, for which the absolute magnitudes based on luminosity distances do not depend on the redshift. It is:

$$
\begin{gathered}
\Omega_{\Lambda}=0.42, \Omega_{M}=0.58 \\
q_{0}=0.18
\end{gathered}
$$



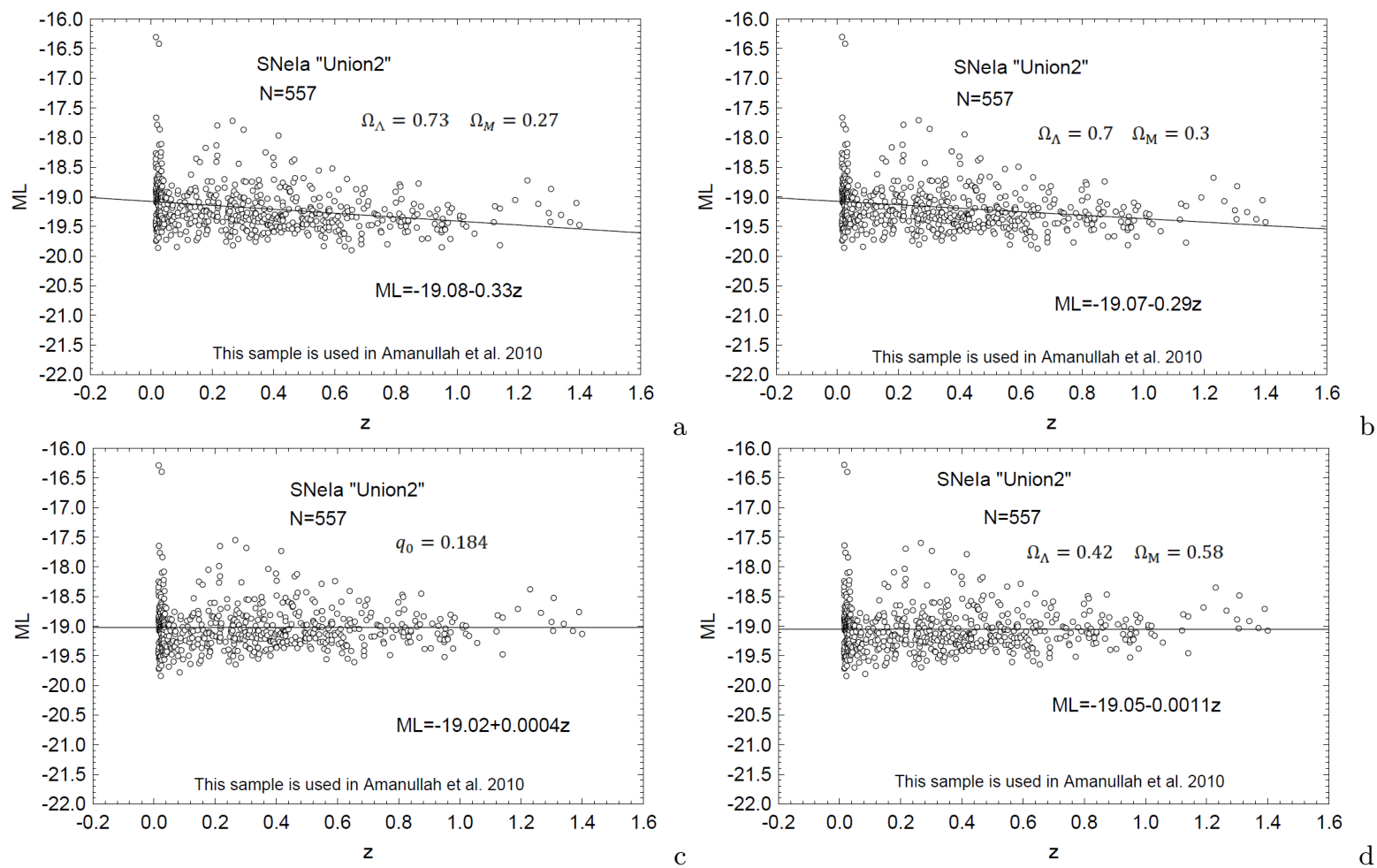

Figure 10. Absolute magnitude dependence on redshift in the sample Amanullah et al. (2010) in the case of various cosmological models.

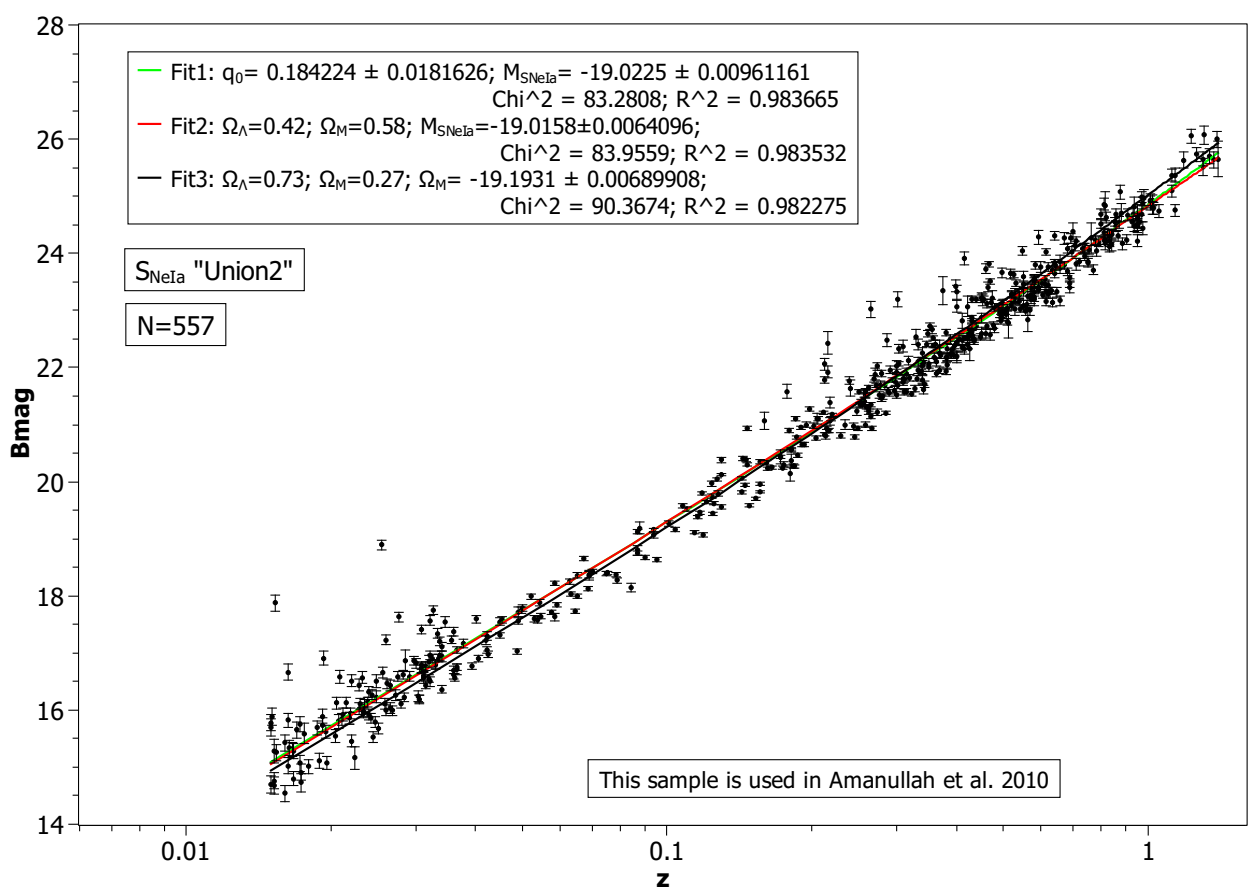

Figure 11. Hubble diagram for Amanullah et al. (2010) sample. 


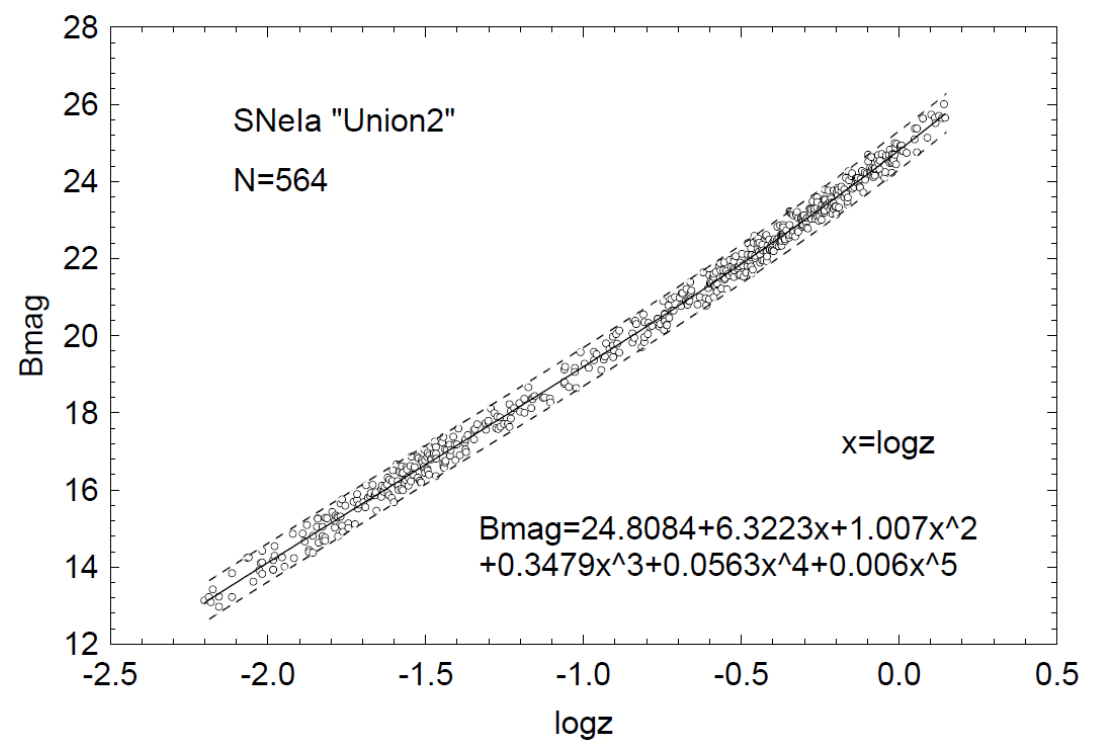

Figure 12. Hubble diagram, formed as a result of "polynomial filtering" of the SNIa "Union2" sample.
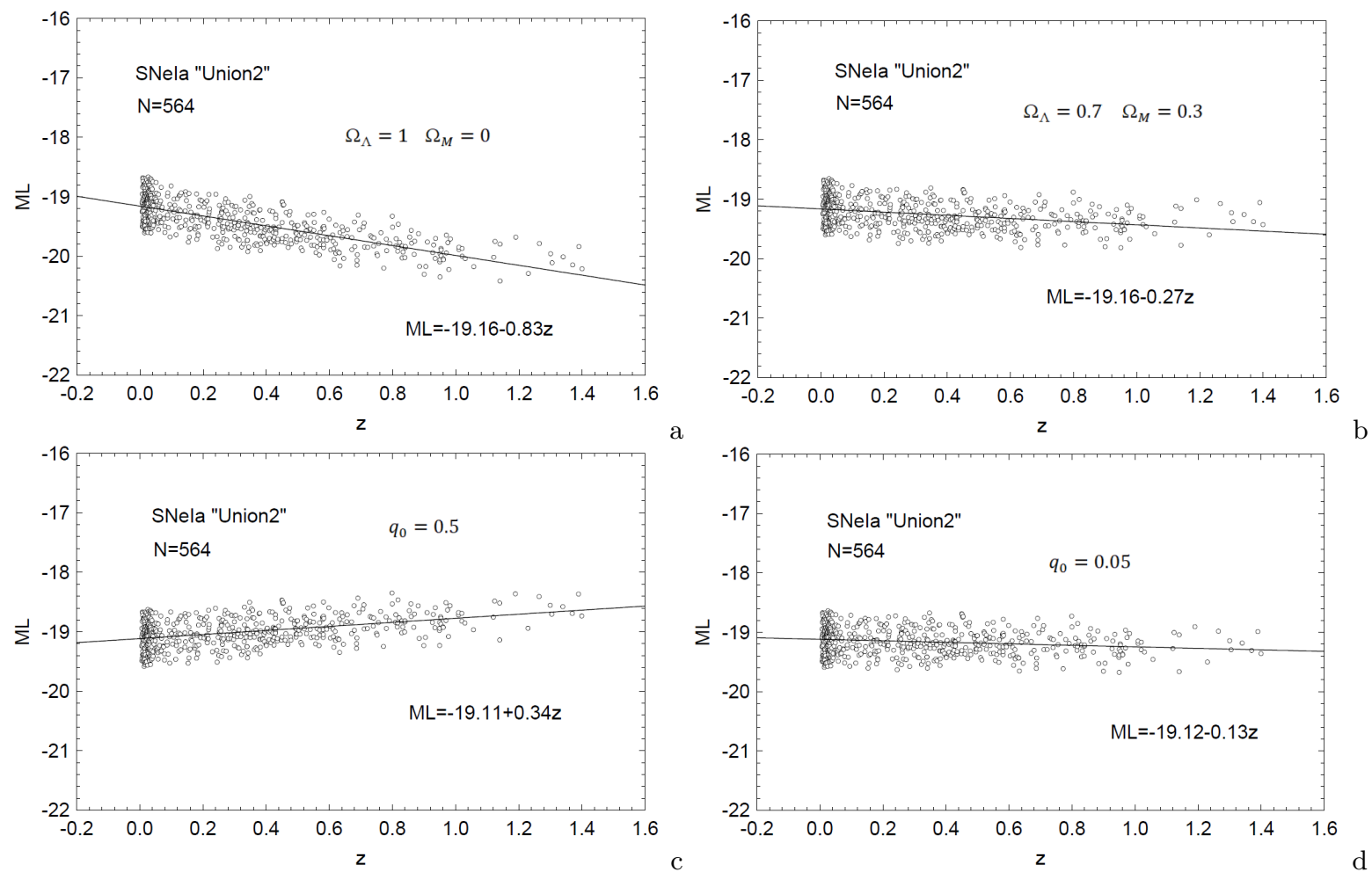

Figure 13. Absolute magnitude - redshift dependence for a sample formed as a result of polynomial filtering for various parameter values in models with $\Omega_{\Lambda}=0$ and $\Omega_{K}=0$. 
Figure 10 shows the corresponding dependences between absolute magnitude and redshift. These dependencies are also given for the cases $\Omega_{\Lambda}=0.73, \Omega_{M}=0.27$ and $\Omega_{\Lambda}=0.7, \Omega_{M}=0.3$. In the latter two cases, the dependence is obvious.

Now consider the Hubble diagram (Figure 11). Here also, the smallest square deviation of the theoretical curve from observation points gives us the model with $\Omega_{\Lambda}=0$. And for the model with $\Omega_{K}=0$, the observations are better represented in the case of $\Omega_{\Lambda}=0.42, \Omega_{M}=0.58$ (when there is no dependence between absolute magnitude and redshift.) than with obtained in Amanullah et al. (2010) values $\Omega_{\Lambda}=0.73, \Omega_{M}=0.27$.

Now we will try to clear the "Union2" sample from stars that for any reason do not meet the definition of the distance indicator.

To avoid dependence on any model as a result of this choice, we use the following technique:

We approximate the Hubble diagram with a polynomial of 5 degrees. Take two parallel curves at a distance of $\Delta m= \pm 0.75$ on either side of the approximated curve. We will remove the stars falling out of this layer. The remaining points we'll approximate with a new polynomial of degree 5 . Take a smaller $\Delta m$ and again construct two curves around this diagram, and remove the points that fall out of the layer. This process will continue to $\Delta m= \pm 0.5$. That is, the width of the Hubble diagram will be one magnitude.

Figure 12 shows the Hubble diagram, obtained in this way for 564 stars,

As above (Figure 2, Figure 6), in Figure 13 shows the dependence between absolute magnitude and redshift for the following cases:

a. $\Omega_{\Lambda}=1, \quad \Omega_{M}=0$

b. $\Omega_{\Lambda}=0.7, \quad \Omega_{M}=0.3$

c. $q_{0}=0.5\left(\right.$ or $\left.\Omega_{M}=1\right)$

d. $q_{0}=0.05$ :

In all cases, the dependence between absolute magnitude - redshift is significant.

For the two cosmological models under discussion $\left(\Omega_{\Lambda}=0\right.$ and $\left.\Omega_{K}=0\right)$, Figure 14 shows absolute magnitude - redshift dependence for those values of the parameters under discussion for which there is no dependence. Thus, the $M_{S N e I a} \neq M_{S N e I a}(z)$ test shows that the absolute magnitude of supernovae in the case of the model with $\Omega_{\Lambda}=0$ does not depend on the redshift when $q_{0}=0.152$, and in the case of the model $\left(\Omega_{K}=0\right)$ this result is obtained for $\Omega_{\Lambda}=0.449, \Omega_{M}=0.551$.
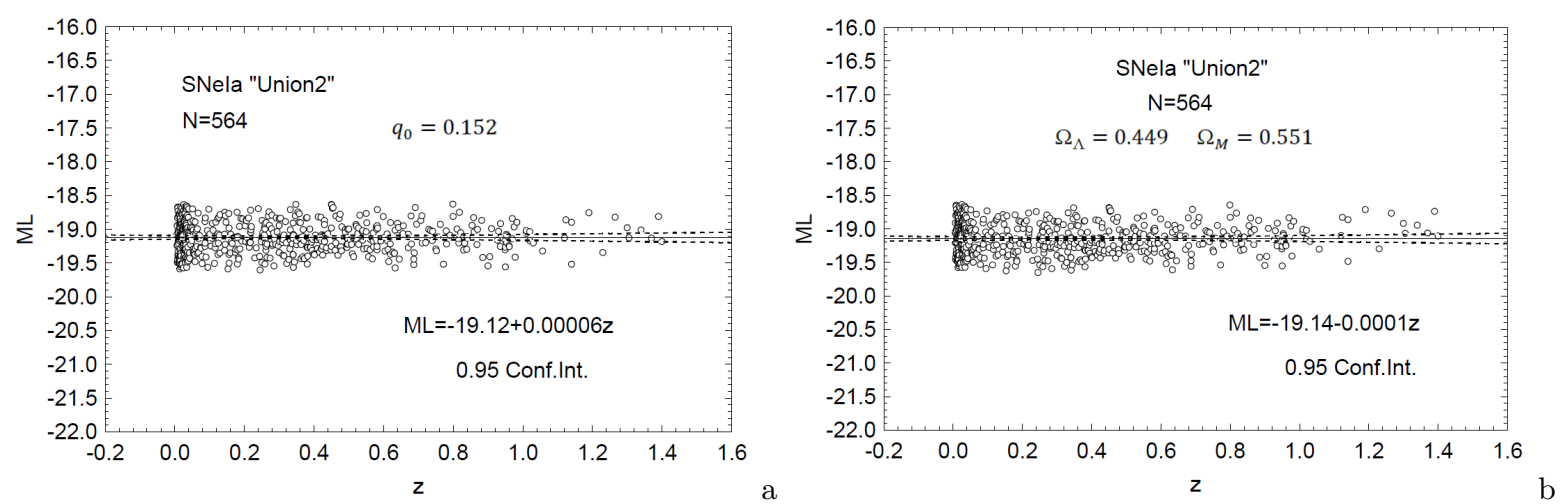

Figure 14. Absolute magnitude - redshift dependence for a sample created as a result of polynomial filtering for the values of the model parameters $\Omega_{\Lambda}=0$ and $\Omega_{K}=0$, satisfying the $M_{\text {SNeIa }} \neq M_{\text {SNeIa }}(z)$ test.

Consider the Hubble diagram, which is shown in Figure $15 \mathrm{a}$. In the case of the $\Omega_{\Lambda}=0$, the approximation gives $q_{0}=0.1518 \pm 0.0058, M_{S N I a}=-19.119 \pm 0.003$, and in the case of the model $\Omega_{K}=0$, by fixing the values obtained from the $M_{S N e I a} \neq M_{S N e I a}(z), \Omega_{\Lambda}=0.449, \Omega_{M}=0.551$, after approximation we get $M_{S N \text { Ia }}=-19.158 \pm 0.002$, and for a fixed value $\Omega_{\Lambda}=0.7, \Omega_{M}=0.3$ we get $M_{S N e I a}=-19.249 \pm 0.003$. The sum of the squares of deviations from the theoretical curve is the smallest in the case of the model of $\Omega_{\Lambda}=0\left(C h i^{2}=28.6\right)$. In the case of a model for a flat universe, these values are respectively the following: $\left(\Omega_{\Lambda}=0.449, \Omega_{M}=0.551\right)-C h i^{2}=29.4,\left(\Omega_{\Lambda}=0.7, \Omega_{M}=0.3\right)$ - 
$C h i^{2}=33.8:$ In other words, the values $\left(\Omega_{\Lambda}=0.449, \Omega_{M}=0.551\right)$ in the case of a model with $\Omega_{K}=0$ better describe the universe than the values $\Omega_{\Lambda}=0.7, \Omega_{M}=0.3$.

In Figure 15 for the indicated models, the dependences of the residues on the redshift are also shown (Figure 15b, c, d). These graphs and the values of $C h i^{2}$ given in them also show the advantage of the model with zero cosmological constant.
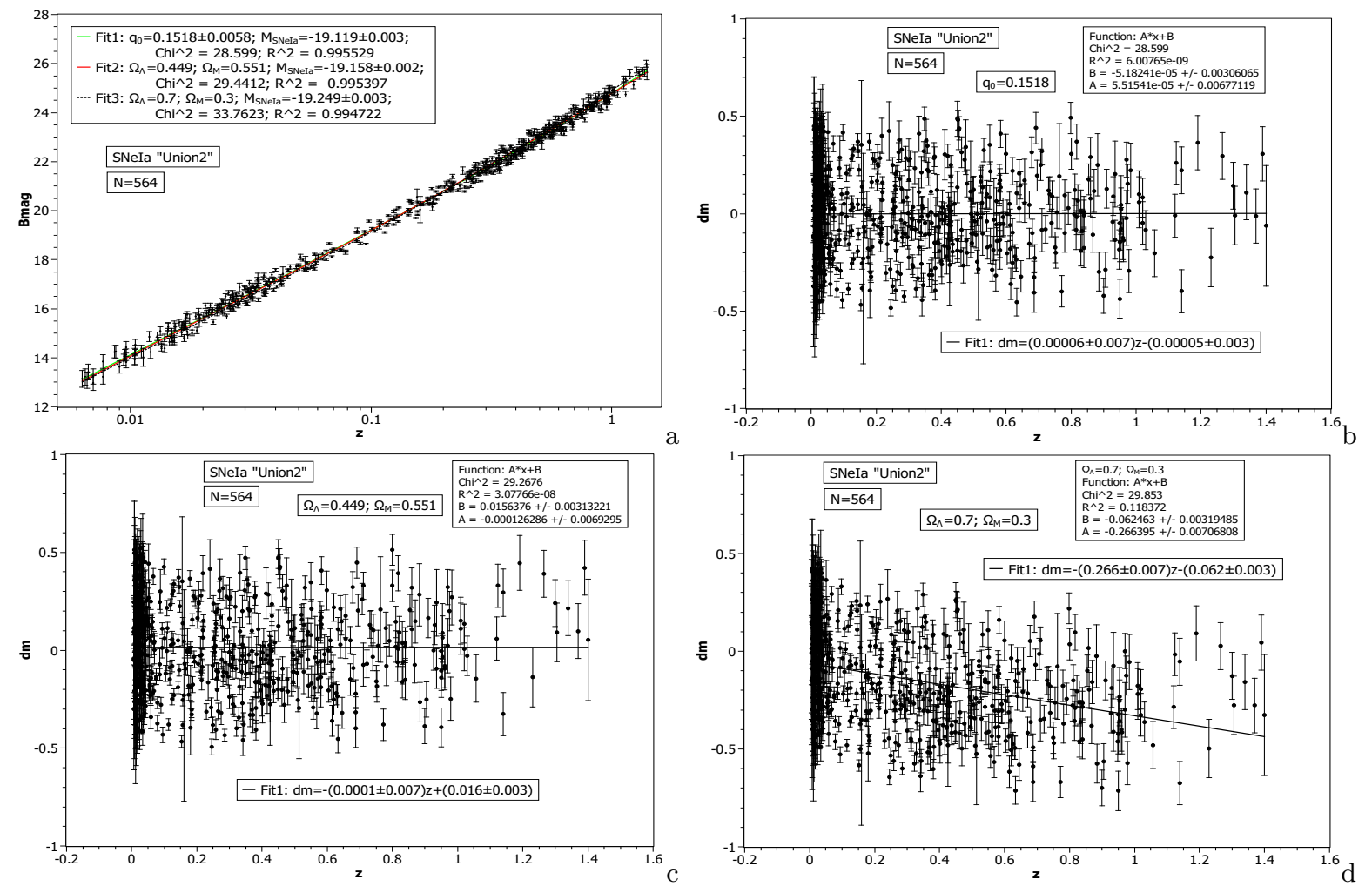

Figure 15. Hubble diagram (a) and the dependence of residuals on the redshift for the sample obtained as a result of polynomial filtering ( $\mathrm{b}-q_{0}=0.1518, \mathrm{c}-\Omega_{\Lambda}=0.449, \Omega_{M}=0.551, \mathrm{~d}-\Omega_{\Lambda}=0.7, \Omega_{M}=0.3$ ).

The same applies to the statistics of $d m$ and $d m 2$ values, which are shown in Tables 1 and 2 . The same shows the statistics of these values for different redshift ranges (Tables 3 and 4).

Table 1. Statistics of dm deviations from theoretical curves for different models

\begin{tabular}{lllllllll}
\hline Variable $d m$ & $\mathrm{~N}$ & Mean & Conf. -95\% & Conf. 95\% & Median & Variance & Std. Dev. & Std. Err. \\
\hline$q_{0}=0.1518$ & 564 & -0.000 & -0.019 & 0.019 & -0.005 & 0.051 & 0.225 & 0.009 \\
$\Omega_{\Lambda}=0.449, \Omega_{M}=0.551$ & 564 & 0.016 & -0.003 & 0.034 & 0.009 & 0.052 & 0.228 & 0.010 \\
$\Omega_{\Lambda}=0.7, \Omega_{M}=0.3$ & 564 & 0.001 & -0.020 & 0.021 & -0.024 & 0.060 & 0.245 & 0.010 \\
\hline
\end{tabular}

Thus, there are at least two reasons to give an advantage to the model of $\Omega_{\Lambda}=0$. They are:

a. The Hubble diagram is more accurately represented by a theoretical curve in the case of the model of $\Omega_{\Lambda}=0$,

b. There is no need to introduce the concept of dark energy.

Even if we assume that dark energy exists, it cannot be overwhelming, because the value of $\Omega_{\Lambda}$ is 0.45 when using the model of $\Omega_{K}=0$. 
Table 2. Statistics of $\mathrm{dm}^{2}$ deviations from theoretical curves for different models

\begin{tabular}{lllllllll}
\hline Variable $d m^{2}$ & $\mathrm{~N}$ & Mean & Conf -95\% & Conf 95\% & Median & Variance & Std. Dev. & Std. Err. \\
\hline$q_{0}=0.1518$ & 564 & 0.051 & 0.046 & 0.055 & 0.028 & 0.003 & 0.057 & 0.002 \\
$\Omega_{\Lambda}=0.449, \Omega_{M}=0.551$ & 564 & 0.052 & 0.047 & 0.057 & 0.027 & 0.003 & 0.059 & 0.002 \\
$\Omega_{\Lambda}=0.7, \Omega_{M}=0.3$ & 564 & 0.060 & 0.054 & 0.066 & 0.035 & 0.005 & 0.071 & 0.003 \\
\hline
\end{tabular}

Table 3. Statistics of dm deviations from theoretical curves for different models and different redshift intervals

\begin{tabular}{|c|c|c|c|c|c|c|c|c|}
\hline Variable dm & $\mathrm{z}$ & $\mathrm{N}$ & Mean & Conf. $-95 \%$ & Conf. $95 \%$ & Median & Variance & Std. Err. \\
\hline$q_{0}=0.1518$ & $0.00-0.25$ & 280 & 0.002 & -0.026 & 0.031 & 0.018 & 0.058 & 0.014 \\
\hline$\Omega_{\Lambda}=0.449, \Omega_{M}=0.551$ & & & 0.029 & 0.000 & 0.057 & 0.038 & 0.059 & 0.014 \\
\hline$q_{0}=0.1518$ & $0.251-0.45$ & 119 & -0.017 & -0.055 & 0.022 & -0.034 & 0.045 & 0.019 \\
\hline$\Omega_{\Lambda}=0.449, \Omega_{M}=0.551$ & & & -0.029 & -0.067 & 0.010 & -0.047 & 0.045 & 0.019 \\
\hline$q_{0}=0.1518$ & $0.451-0.65$ & 77 & 0.002 & -0.046 & 0.050 & -0.019 & 0.044 & 0.024 \\
\hline$\Omega_{\Lambda}=0.449, \Omega_{M}=0.551$ & & & -0.003 & -0.050 & 0.044 & -0.026 & 0.043 & 0.024 \\
\hline$q_{0}=0.1518$ & $0.651-0.85$ & 41 & 0.023 & -0.044 & 0.089 & 0.010 & 0.044 & 0.033 \\
\hline$\Omega_{\Lambda}=0.449, \Omega_{M}=0.551$ & & & 0.039 & -0.028 & 0.105 & 0.037 & 0.044 & 0.033 \\
\hline$q_{0}=0.1518$ & $0.851-1.05$ & 33 & -0.018 & -0.087 & 0.050 & -0.023 & 0.037 & 0.033 \\
\hline$\Omega_{\Lambda}=0.449, \Omega_{M}=0.551$ & & & 0.023 & -0.045 & 0.092 & 0.023 & 0.037 & 0.033 \\
\hline$q_{0}=0.1518$ & $1.051-1.45$ & 14 & 0.056 & -0.076 & 0.187 & 0.050 & 0.052 & 0.061 \\
\hline$\Omega_{\Lambda}=0.449, \Omega_{M}=0.551$ & & & 0.145 & 0.010 & 0.279 & 0.156 & 0.054 & 0.062 \\
\hline
\end{tabular}

Table 4. Statistics of $\mathrm{dm}^{2}$ deviations from theoretical curves for different models and different redshift intervals

\begin{tabular}{lllllllll}
\hline Variable $\mathrm{dm}^{2}$ & $\mathrm{z}$ & $\mathrm{N}$ & Mean & Conf. -95\% & Conf. 95\% & \multicolumn{2}{c}{ Median Variance } & Std. Err. \\
\hline$q_{0}=0.1518$ & $0.00-0.25$ & 280 & 0.058 & 0.051 & 0.065 & 0.036 & 0.004 & 0.004 \\
$\Omega_{\Lambda}=0.449, \Omega_{M}=0.551$ & & & 0.059 & 0.052 & 0.067 & 0.036 & 0.004 & 0.004 \\
\hline$q_{0}=0.1518$ & $0.251-0.45$ & 119 & 0.045 & 0.035 & 0.055 & 0.023 & 0.003 & 0.005 \\
$\Omega_{\Lambda}=0.449, \Omega_{M}=0.551$ & & & 0.046 & 0.036 & 0.055 & 0.026 & 0.003 & 0.005 \\
\hline$q_{0}=0.1518$ & $0.451-0.65$ & 77 & 0.043 & 0.031 & 0.055 & 0.023 & 0.003 & 0.006 \\
$\Omega_{\Lambda}=0.449, \Omega_{M}=0.551$ & & & 0.043 & 0.031 & 0.055 & 0.022 & 0.003 & 0.006 \\
\hline$q_{0}=0.1518$ & $0.651-0.85$ & 41 & 0.044 & 0.025 & 0.063 & 0.014 & 0.004 & 0.009 \\
$\Omega_{\Lambda}=0.449, \Omega_{M}=0.551$ & & & 0.045 & 0.025 & 0.065 & 0.017 & 0.004 & 0.010 \\
\hline$q_{0}=0.1518$ & $0.851-1.05$ & 33 & 0.036 & 0.020 & 0.053 & 0.016 & 0.002 & 0.008 \\
$\Omega_{\Lambda}=0.449, \Omega_{M}=0.551$ & & & 0.037 & 0.021 & 0.052 & 0.018 & 0.002 & 0.007 \\
\hline$q_{0}=0.1518$ & $1.051-1.45$ & 14 & 0.056 & -0.076 & 0.187 & 0.050 & 0.052 & 0.061 \\
$\Omega_{\Lambda}=0.449, \Omega_{M}=0.551$ & & & 0.071 & 0.032 & 0.110 & 0.052 & 0.005 & 0.018 \\
\hline
\end{tabular}


Thus, we can conclude that we are in an infinite expanding Universe, filled only with the matter with gravitational interaction, the density of which is $\sim 30 \%$ of the critical density. We can safely say that cosmological models do not need an assumption about dark energy. We can conclude that the Universe consists of luminous and dark matter.

If we exclude the stars that are in Amanullah et al. (2010) and considered "bad" stars, indicated in the "Fail" column in their Table. 2, then 479 stars will remain in the sample with which the following values can be obtained using the same procedure:

$$
\begin{gathered}
q_{0}=0.134 \pm 0.006 ; M_{S N e I a}=-19.13 \pm 0.003 ; C h i^{2}=23.596 \\
\Omega_{\Lambda}=0.486, \Omega_{M}=0.514 ; M_{S N e I a}=-19.17 \pm 0.002 ; C h i^{2}=24.285
\end{gathered}
$$

Figure 16 shows the Hubble diagram for this case. Here the model $\Omega_{\Lambda}=0$ also has an advantage over the model with $\Omega_{K}=0$.



Figure 16. Hubble diagram for the sample after polynomial filtering and exclusion of "Fail" stars.

\section{Discussion}

Thus, it turns out that in the case of the two discussed models $\left(\Omega_{\Lambda}=0\right.$ and $\Omega_{K}=0$ ), there are $q_{0}$ and $\Omega_{\Lambda}, \Omega_{M}$ values for which we obtain the absolute magnitude, which is estimated from the luminosity distance from the corresponding cosmological model, which does not depend on redshift. It is with these values that these models best represent the Hubble diagram. It should be noted that the mathematical analysis shows that a model with a cosmological constant of zero describes the Universe better than a model for a flat universe, but in both cases, the accuracy is quite high, and the results are quite close. So, trusting only mathematics, in this case, is not enough. The result depends largely on the observations, the accuracy of their processing, as well as on a sample of supernovae. In Figure 17, for a sample formed 
as a result of polynomial filtering, we give d_m depending on the redshift value, where

$$
d_{m}=5 \log \left[\frac{D_{L}\left(\Omega_{L}=0.449, \Omega_{M}=0.551\right)}{D_{L}\left(q_{0}=0.151762\right)}\right]
$$

is the difference in apparent magnitudes derived from these two models. In the case of small $z(z<1)$, the maximum difference falls on $z=0.364$ and is 0.053 . For $z=1$, this difference is almost equal to 0 $\left(d \_m=-0,011\right)$. For $z=1.4$, where our observed data end, $d \_m=-0.076$. This means that both the accuracy of modern observations and a specific sample of supernovae can have a significant impact on the final conclusion. This difference becomes more or less significant at $z=2.0 \div 2.5\left(d \_m=-0.175 \div-0.251\right)$. So, for the final conclusion, we need reliable observational material at least up to redshifts $z=2.5$. This means that our observational technology should be able to detect and process objects with an apparent magnitude about 1.5 magnitudes weaker than currently observed.

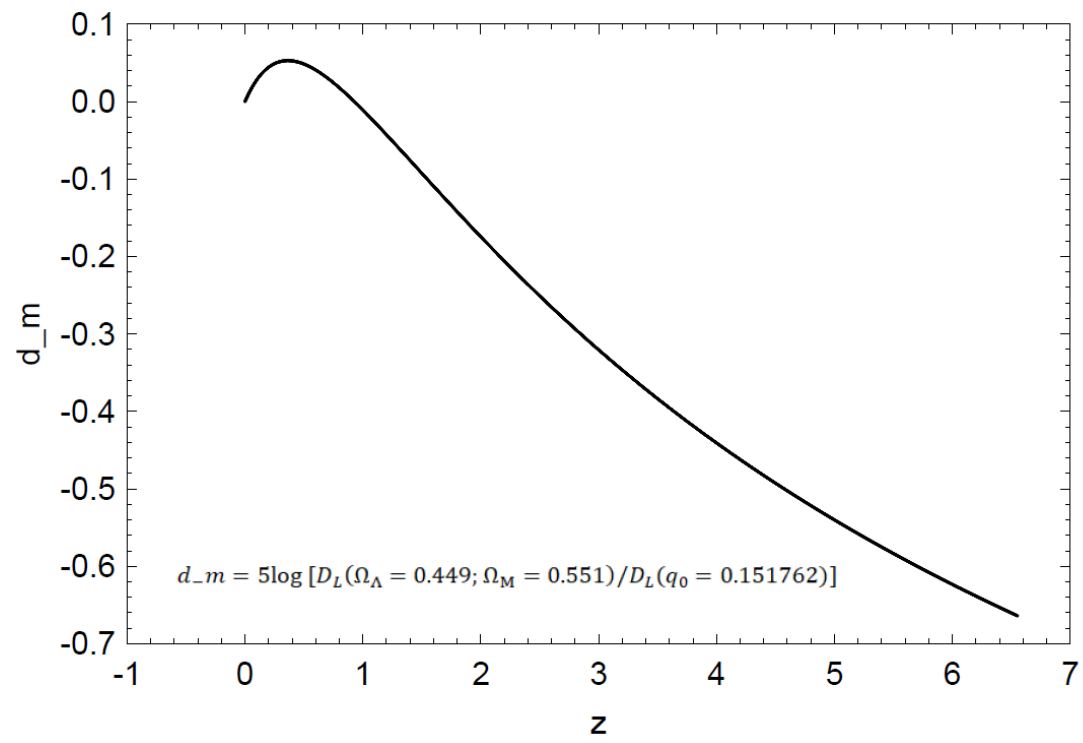

Figure 17. Dependence of $d_{m}=5 \log \left[\frac{D_{L}\left(\Omega_{L}=0.449, \Omega_{M}=0.551\right)}{D_{L}\left(q_{0}=0.151762\right)}\right]$ on the redshift for the sample obtained by the "polynomial filtering" method.

In any case, the result obtained in this work leads to the idea that modern observational material allows us to conclude that a model with a cosmological constant of zero represents the Universe better than a model for a flat, $\Lambda C D M$ universe. It also allows us to refrain from the idea of introducing a hypothetical substance, such as "dark energy."

\section{Conclusions}

On the basis of samples of supernovae SNe Ia "Union" and "Union2" in this paper we discuss two models describing the Universe. One of them (the model with a cosmological constant with $\Omega_{\Lambda}=0$ was widespread until 1998, when the study of type Ia supernovae showed that the Universe is expanding at an accelerating rate. After these studies, the model for a flat universe became dominant and was also considered in this paper.

In order to accept or reject a particular cosmological model, it is proposed to use the absolute magnitude test, based on the generally accepted fact that the absolute magnitude of type Ia supernovae is almost constant and that this value calculated using theoretically obtained luminosity distances for a particular model should not depend upon the redshift i.e. $M_{S N e I a} \neq M_{S N e I a}(z)$. 
In this paper, we studied different samples of the compilation of SNeIa Union and SNeIa Union2:

1. This sample includes all the stars of SNeIa Union with magnitude data, except for 1996ai.

2. A sample of Kowalski et al. (2010).

3. All stars with magnitude data of the SNeIa Union2 sample are included, with the exception of 1996ai, 2003fa, 2005ew, 2006bk, 2006mq stars, which are clearly far from the general pattern on the Hubble diagram.

4. Exactly the sample that was used in Amanullah et al. (2010).

5. Stars with a large deviation from the Hubble diagram were removed from the SNeIa Union2 sample. The procedure for excluding stars is described above.

6. From sample 5, the stars that are in Amanullah et al. (2010) are listed in the "Fail" column are also removed.

For these samples, we found the values of model parameters for which the absolute magnitudes of supernovae stars obtained from the luminosity distances of the corresponding models do not depend on the redshift. It turns out that in the case of the values of these parameters, the Hubble diagram is best approximated by a theoretical curve.

Table 5 shows the values of the parameters at which the models are best consistent with observational data.

Table 5. The values of the parameters of cosmological models for which observational data are best consistent with theory

\begin{tabular}{|c|c|c|c|c|c|c|c|c|c|}
\hline & Sample & $q_{0}$ & $\begin{array}{l}M_{S N e I a} \\
\left(q_{0}\right)\end{array}$ & $C h i^{2}$ & $\Omega_{\Lambda}$ & $\Omega_{M}$ & $\begin{array}{l}M_{S N e I a} \\
\left(\Omega_{\Lambda}, \Omega_{M}\right)\end{array}$ & $\begin{array}{l}q_{0} \\
\left(\Omega_{\Lambda}, \Omega_{M}\right)\end{array}$ & $C h i^{2}$ \\
\hline 1 & $\begin{array}{l}\text { Union } \\
\mathrm{N}=397\end{array}$ & $\begin{array}{l}0.310 \\
\pm 0.032\end{array}$ & $\begin{array}{l}-18.89 \\
\pm 0.018\end{array}$ & 83.85 & 0.255 & 0.745 & $\begin{array}{l}-18.90 \\
\pm 0.011\end{array}$ & +0.12 & 84.86 \\
\hline 2 & $\begin{array}{l}\text { Union } \\
\mathrm{N}=307^{*}\end{array}$ & $\begin{array}{l}0.143 \\
\pm 0.018\end{array}$ & $\begin{array}{l}-19.01 \\
\pm 0.012\end{array}$ & 35.51 & 0.501 & 0.499 & $\begin{array}{l}-19.06 \\
\pm 0.007\end{array}$ & -0.25 & 35.14 \\
\hline 3 & $\begin{array}{l}\text { Union2 } \\
\mathrm{N}=680\end{array}$ & $\begin{array}{l}0.268 \\
\pm 0.0 .2\end{array}$ & $\begin{array}{l}-18.96 \\
\pm 0.012\end{array}$ & 150.3 & 0.30 & 0.70 & $\begin{array}{l}-18.98 \\
\pm 0.009\end{array}$ & +0.05 & 152.5 \\
\hline 4 & $\begin{array}{l}\text { Union2 } \\
\mathrm{N}=557^{* *}\end{array}$ & $\begin{array}{l}0.184 \\
\pm 0.018\end{array}$ & $\begin{array}{l}-19.02 \\
\pm 0.010\end{array}$ & 83.28 & 0.42 & 0.58 & $\begin{array}{l}-19.05 \\
\pm 0.006\end{array}$ & -0.13 & 83.96 \\
\hline 5 & $\begin{array}{l}\text { Union2 } \\
\mathrm{N}=564\end{array}$ & $\begin{array}{l}0.152 \\
\pm 0.006\end{array}$ & $\begin{array}{l}-19.12 \\
\pm 0.003\end{array}$ & 28.60 & 0.449 & 0.551 & $\begin{array}{l}-19.16 \\
\pm 0.002\end{array}$ & -0.17 & 29.44 \\
\hline 6 & $\begin{array}{l}\text { Union2 } \\
\mathrm{N}=479\end{array}$ & $\begin{array}{l}0.134 \\
\pm 0.006\end{array}$ & $\begin{array}{l}-19.13 \\
\pm 0.003\end{array}$ & 23.60 & 0.486 & 0.514 & $\begin{array}{l}-19.17 \\
\pm 0.002\end{array}$ & -0.23 & 24.29 \\
\hline
\end{tabular}

* This sample is used in Kowalski et al. 2008

** This sample is used in Amanullah et al. 2010

It should also be noted that the values of these parameters in the model for a flat, $\Lambda C D M$ universe are quite far from the generally accepted values. Also not confirmed, the generally accepted "superiority of energy". Within this model, the priority of the mass is obtained. Even in two cases (samples 1 and 3 ) the cosmological deceleration parameter $q_{0}=1 / 2\left(\Omega_{M}-2 \Omega_{\Lambda}\right)$ obtained from the model is positive, that is, the Universe expands with deceleration.

As for the model with the zero cosmological constant $\left(\Omega_{\Lambda}=0\right)$, it represents the observational data better than the model for a flat universe. Only in one of the six cases (sample 2) the sum of the deviations of the observation points from the theoretical curve is somewhat larger than in the model with $\Omega_{K}=0$. Note that sample 2 is the smallest.

Thus, we must conclude that the expansion of the Universe with acceleration does not correspond to reality. Consequently, the idea of dark energy, widespread in the scientific literature, is unacceptable.

In the universe, there is a mass superiority and when calculating distances to extragalactic objects, at least to the redshift $z \sim 1.5$, formula (6) derived from the model with a cosmological constant of zero should be used. 
The universe is open and essentially consists of matter with a density of $\sim(26-36) \%$ of the critical density. This number is derived from a study of relatively reliable 4, 5, 6 samples and coincides with the accepted total amount of visible and dark matter.

\section{References}

1. R. Amanullah et al. 2008, A\&A, 486, 375

2. R. Amanullah et al. 2010, ApJ, 716, 712

3. P. Astier et al. 2006, A\&A, 447, 31

4. B. Barris et al. 2004, ApJ, 602, 571

5. S. M. Carroll, W. H. Press \& E. L. Turner 1992, ARA\&A, 30, 499

6. W. A. Fowler \& F. Hoyle 1960, ApJ, 132, 565

7. P. Garnavich et al. 1998, ApJ, 509, 74

8. J. Guillochon, J. Parrent, L. Z. Kelley \& R. Margutti 2017, ApJ, 835, 1. https://sne.space/

9. M. Hamuy et al. 1996a, AJ, 112, 2398

10. M. Hamuy et al. 1996b, AJ, 112, 2408

11. M. Hicken et al. 2009a, ApJ, 700, 331

12. M. Hicken et al. 2009b, ApJ, 700, 1097

13. J. A. Holtzman et al. 2008, AJ, 136, 2306

14. E. P. Hubble, Proc. Nat. Acad. Sci. 1929, 15, 168

15. S. Jha et al. 2006, AJ, 131, 527

16. R. Knop et al. 2003, ApJ, 598, 102

17. M. Kowalski et al. 2008, ApJ, 686, 749

18. K. Krisciunas et al. 2004a, AJ, 127, 1664

19. K. Krisciunas et al. 2004b, AJ, 128, 3034

20. K. Krisciunas et al. 2005, AJ, 130, 2453

21. W. Mattig 1958, Astron. Nachr. 284, 109

22. G. Miknaitis et al. 2007, ApJ, 666, 674

23. S. Perlmutter et al. 1999, ApJ, 517, 565

24. A. Riess et al. 1998, AJ, 116, 1009

25. A. Riess et al. 1999, AJ, 117, 707

26. A. Riess et al. 2007, ApJ, 659, 98

27. A. Sandage \& G.Tammann 1982, ApJ, 256, 339

28. B. Schmidt et al. 1998, ApJ, 507, 46

29. V. M. Slipher, table prepared for book of A. S. Eddington, The Mathematical Theory of Relativity, 2nd ed. (Cambridge University Press, London, 1924): 162.

30. J. Tonry et al. 2003, ApJ, 594, 1

31. S. Weinberg, Cosmology, Oxford University Press, 2008 